\title{
Patterns of seroconversion for SARS-CoV-2 lgG in patients with malignant disease and association with anticancer therapy
}

\author{
Astha Thakkar ${ }^{1}$, Kith Pradhan², Shawn Jindal ${ }^{3}$, Zhu Cui ${ }^{3}$, Bradley Rockwell ${ }^{3}$, Akash Pradip Shah', \\ Stuart Packer ${ }^{1}$, R. Alejandro Sica', Joseph Sparano', D. Yitzhak Goldstein ${ }^{10}{ }^{4}$, Amit Verma', \\ Sanjay Goel ${ }^{1 \times}$ and Balazs Halmos ${ }^{1 \times}{ }^{1 凶}$
}

Patients with cancer have been identified in several studies to be at high risk of developing severe COVID-19; however, rates of SARS-CoV-2 IgG seroconversion and its association with cancer types and anticancer therapy remain obscure. We conducted a retrospective cohort study in patients with cancer who underwent SARS-CoV-2 lgG testing. Two hundred and sixty-one patients with a cancer diagnosis underwent SARS-CoV-2 IgG testing and demonstrated a high rate of seroconversion (92\%). However, significantly lower seroconversion was observed in patients with hematological malignancies (82\%), patients who received anti-CD-20 antibody therapy (59\%) and stem cell transplant (60\%). Notably, all 17 patients who received immunotherapy, including 16 that received anti-PD-1/PD-L1 monoclonal antibodies, developed SARS-CoV-2 IgG antibodies (100\% seroconversion). These data show differential rates of seroconversion in specific patient groups and bear importance for clinical monitoring and vaccination strategies that are being developed to mitigate the COVID-19 pandemic.

\begin{abstract}
T he coronavirus pandemic that started in December 2019 in Wuhan, China continues to send waves of COVID-19 disease throughout the world ${ }^{1,2}$. Several observational studies have identified patients with cancer as being at higher risk of contracting the virus with higher rates of manifesting a severe form of COVID19 disease $^{3-5}$. We have previously reported a higher case fatality rate in patients with hematological malignancies compared to solid malignancies in patients with cancer ${ }^{6}$. A pooled meta-analysis of 52 studies involving patients with cancer and COVID-19 reported a mortality rate of $25.6 \%{ }^{7}$. While the mortality rates of patients with cancer are higher than the general population, it seems that about $70-80 \%$ of patients with cancer survive COVID-19 and therefore, it is important to understand the natural history of COVID-19 in this high-risk patient population. Of particular importance is the fact that this patient population often receives immunosuppressive cancer-directed therapy, which may impact their ability to mount a humoral immune response to the virus. It is therefore prudent to study the rate of formation of such antibodies to SARS-CoV-2 in patients with cancer who survived the illness to properly inform and develop treatment, surveillance and monitoring strategies in this vulnerable patient population.
\end{abstract}

\section{Results}

Patient selection. We collected data for all patients with a cancer diagnosis cared for at the Montefiore Health System (MHS) starting 1 March 2020 (first observed COVID-19 infection at MHS) until 15 September 2020. Figure 1 represents cohort selection for this study. A total of 4,302 patients were identified, of which 3,562 were excluded as they did not have a SARS-CoV-2 PCR with reverse transcription (RT-PCR) test result in our system, leaving 740 patients. Of the 740 patients, 460 were excluded as 8 patient records were duplicates and 452 did not have a SARS-CoV-2 IgG test. After excluding the aforementioned patients, 280 patients were identified of which, 15 were excluded as they did not have a confirmed diagnosis of malignancy. Three more patients were excluded as they had a negative SARS-CoV-2 PCR and a negative SARS-CoV-2 IgG and one patient was excluded as negative SARS-CoV-2 IgG test preceded a positive SARS-CoV-2 PCR. Finally, 261 patients with a confirmed diagnosis of malignancy and at least one SARS-CoV-2 IgG test performed during their care at MHS were included for analysis.

Baseline characteristics. A total of 261 patients with a confirmed diagnosis of malignancy were included in this study. The median age of the cohort was 64 years (range 20-90 years). Seventy-seven percent (201 of 261) had a diagnosis of solid malignancy and $23 \%$ (60 of 261) had a hematological malignancy. Fifty-one percent (134 of 261) of patients were female and 49\% (127 of 261) were male. Forty-one percent (106 of 261) of patients were AfricanAmerican, 37\% (98 of 261) were Hispanic, 13\% (33 of 261) were white, $3 \%$ (8 of 261) were Asian and 6\% (16 of 261) belonged to other ethnicities.

As expected, we had a preponderance of patients with solid malignancies; 22\% (58 of 261) had breast cancer, 22\%(57 of 261) had genitourinary cancer, $17 \%$ (44 of 261) had gastrointestinal cancer, 9\% (24 of 261) had thoracic and head and neck cancer, $4 \%$ (10 of 261) had gynecological cancer, 2\% (5 of 261) had central nervous system cancer and 1\% (3 of 261) had skin/musculoskeletal cancer. Among patients with hematological malignancies 10\% (26 of 261) had lymphoid disorders, $8 \%$ (20 of 261) had plasma cell disorders and $5 \%$ (14 of 261) had myeloid disorders.

'Division of Oncology, Montefiore Medical Center/Albert Einstein College of Medicine, The Bronx, NY, USA. ${ }^{2}$ Department of Epidemiology and Population Health, Albert Einstein College of Medicine, The Bronx, NY, USA. ${ }^{3}$ Department of Internal Medicine, Montefiore Medical Center/Albert Einstein College of Medicine, The Bronx, NY, USA. ${ }^{4}$ Department of Pathology, Montefiore Medical Center/Albert Einstein College of Medicine, The Bronx, NY, USA.

凶e-mail: sgoel@montefiore.org; bahalmos@montefiore.org 
a

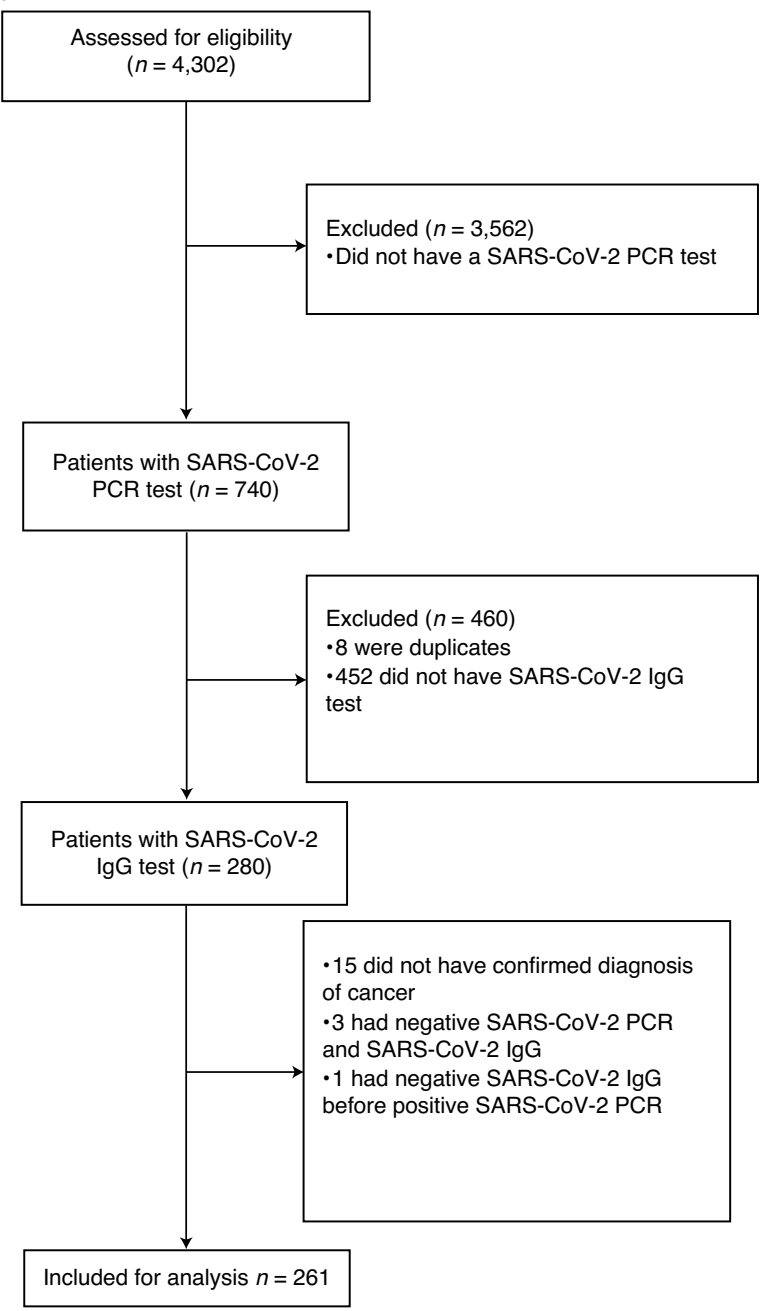

b

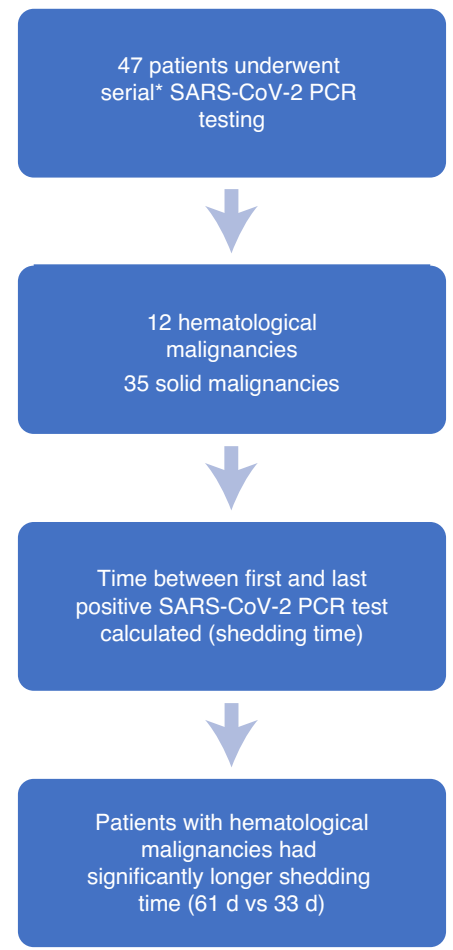

*Patients receiving cancer care at Montefiore Medical Center were required to have a negative SARS-CoV-2 PCR test after a documented COVID-19 infection or exposure and before invasive procedures, in-person visits and before starting or resuming chemotherapy

Fig. 1 | Cohort description and patient inclusion criteria in the present study. a, Consort diagram representing patient selection into the final cohort, listing selection criteria for inclusion into the present study ( $n=$ number of patients at each step). b, Diagram representing patients undergoing serial SARS-CoV-2 PCR testing.

We divided our cohort into patients who had active malignancy within $90 \mathrm{~d}$ of a SARS-CoV-2 test and those who did not. Of 261 patients, $68 \%$ (177 of 261) patients had active malignancy and $32 \%$ (84 of 261) did not. Of the patients with active malignancy, $135 \mathrm{had}$ an initial diagnosis, 23 had progressive disease and 19 had relapsed disease. Of the inactive malignancy subgroup, 71 patients were in remission and 13 patients had malignancy that did not warrant therapy (for example monoclonal gammopathy of unknown significance). Patients were divided into three categories based on their comorbidities, $0-1,2-3$ and $>3$ comorbidities (comorbidities curated by chart review). Cancer diagnosis itself was not included as a comorbidity. The distribution of patients in the comorbidity categories was $26 \%$ (68 of 261), $30 \%$ (78 of 261 ) and $44 \%$ (115 of 261), respectively.

In addition, we also calculated a modified Charlson Comorbidity Index (CCI) for all included patients. Given that malignant conditions comprise 4 out of 19 criteria for the CCI, these were excluded while calculating the CCI for our patients, an approach that has been used in a previous study focused on patients with cancer diagnoses $^{8}$. We then divided the entire cohort into three categories: CCI $0-1,2-3$ and $4+$. The distribution of patients by CCI category is as follows: $0-1,26 \%$ (68 of 261); $2-3,38 \%$ (100 of 261) and $4+, 36 \%$ (93 of 261$)$.
Overall, $92 \%$ of patients (239 of 261) had a positive SARS-CoV-2 IgG test and $8 \%$ (22 of 261) of patients had a negative SARS-CoV-2 IgG test. Fifty-six percent (147 of 261) had symptomatic SARS-CoV-2 infection while $44 \%$ (114 of 261) of patients had an asymptomatic infection. Symptomatic infection rate was $53 \%$ (106 of 201) among patients with solid malignancies and $68 \%$ (41 of 60) among those with hematological malignancies. There was a significant association seen between patients with hematological malignancy and symptomatic infection compared to solid malignancies $(P=0.04)$.

Twenty-three percent of patients (61 of 261) had steroid use at baseline. Of these, 21 patients were on steroids daily and 40 patients received steroids occasionally. The indications and frequencies of steroid use are available in the supplement. The median time between SARS-CoV-2 PCR and SARS-CoV-2 IgG test was $40 \mathrm{~d}$ and mean was $46 \mathrm{~d}$.

Baseline characteristics and frequencies of asymptomatic infection of the cohort are summarized in Table 1 and Supplementary Table 2, respectively.

Cancer treatment history. We collected data for all cancer treatment that was received by each patient. We classified the treatments into the following categories: chemotherapy, immunotherapy, 
Table 1 | Baseline characteristics

\section{Baseline characteristics}

Total number of patients

Median age (range)

Sex

Male

Female

Comorbidities

$0-1$

$2-3$

$>3$

$\mathrm{CCl}$

$0-1$

2-3

$>3$

Ethnicity

African-American

Hispanic

White

Asian

Other

Type of cancer

Solid

Breast cancer

Gastrointestinal cancer

Thoracic/head and neck cancers

Central nervous system cancers

Genitourinary cancer

Gynecological cancer

Skin/musculoskeletal cancer

Hematological

Lymphoid malignancy

Plasma cell malignancy

Myeloid malignancy

SARS-CoV-2 IgG

SARS-CoV-2 IgG positive

SARS-CoV-2 IgG negative

Active cancer

Active

Inactive

Cancer status

Initial diagnosis

Remission

Progressive disease

Relapse

Inactive

Active cancer by cancer group

Solid malignancy

Hematological malignancy

Active cancer treatment within $90 \mathrm{~d}$

Hematological malignancy

Baseline steroid use
Solid malignancy

\section{1}

64 years $(20-90$

years)

n

127

134

n

68

78

115

$n$ tyrosine kinase inhibitors, anti-HER therapy, antibody-drug conjugate, anti-CD20 antibody, anti-CD38 antibody, proteasome inhibitors, immunomodulator, BTK inhibitor, IDH1 inhibitor, BCL2 inhibitor, mTOR inhibitors, PARP inhibitor, TGF- $\beta$ inhibitor, AR-targeted therapy, bispecific-T-cell-engager (BiTE) therapy, anti-EGFR monoclonal antibody, anti-VEGF monoclonal antibody therapy and history of stem cell transplant and CAR-T and cellular therapy. If a patient received two agents falling in the same category, they were classified only once (for example, if a patient received both degarelix and leuprolide, we classified them once in 'endocrine therapy'). Combination and sequential treatment was classified in the appropriate category (for example, a patient receiving rituximab-based chemoimmunotherapy would be classified as received anti-CD-20 antibody, chemotherapy and steroids). CAR-T and cellular therapy included two patients who received CAR-T-cell therapy and one patient who received sipuleucel-T for prostate cancer. The most common treatment modality was cytotoxic chemotherapy in $46 \%$ (119 of 261) of patients followed by endocrine therapy in $27 \%$ (71 of 261) of patients. In the $90 \mathrm{~d}$ preceding a SARS-CoV-2 test, 110 patients had received medical cancer treatment, including 89 patients with a solid malignancy and 21 patients with a hematological malignancy. The frequencies of all treatments and treatments within $90 \mathrm{~d}$ of a COVID test have been summarized in Table 2. The median lines of therapy in the seropositive and seronegative cohorts is 1 .

Clinical course of patients with absent seroconversion. All 22 patients who had a negative SARS-CoV-2 IgG had a preceding SARS-CoV-2 PCR that was positive. Sixteen of 22 patients had symptomatic infections, whereas 6 were asymptomatic. In the symptomatic subgroup, 11 patients were treated on a general medical floor, 1 patient needed intensive care unit (ICU) level of care, 3 patients were quarantined at home and details of the treatment setting for 1 patient are unknown. In the asymptomatic group, one patient was on the general medical floor for a different acute issue, one patient was transferred to ICU for close observation despite no symptoms, one patient was quarantined at home and details of three patients' treatment settings are not available. Overall, in the seronegative cohort of patients, we observed a high symptomatic infection rate and high rates of hospitalization, with some needing ICU level of care.

Eleven of 22 patients had a hematological malignancy and 11 had a solid malignancy. In the seronegative group, 14 patients had chemotherapy, 7 had received anti-CD-20 antibody, 4 had received stem cell transplant, 3 had received a tyrosine kinase inhibitor, 2 patients each had received BiTE and CAR-T and 1 patient each had received an immunomodulator, proteasome inhibitor, antibodydrug conjugate, PARP inhibitor and BTK inhibitor. These treatments are summarized in Supplementary Table 3.

Association between seroconversion and cancer type. Given that patients with hematological malignancies tend to be more immunosuppressed and as several series have suggested, carry higher morbidity with COVID-19, we wanted to investigate differences in seroconversion in patients with hematological versus solid malignancies. Among 60 patients with hematological malignancies, 49 (81.7\%) manifested SARS-CoV-2 IgG positivity, whereas 190 of 201 (94.5\%) patients with solid malignancy manifested SARS-CoV-2 IgG positivity (Fisher's exact test odds ratio $(\mathrm{OR}) 3.8 ; P=0.005)$. Taken together, in our cohort, patients with hematological malignancies had a higher frequency of manifesting symptomatic COVID-19 infection and significantly lower likelihood of seroconversion.

Association between seroconversion and cancer therapy. Furthermore, we aimed to investigate whether seroconversion was associated with the type of cancer therapy received by a patient. In 


\section{Table 2 | Cancer-directed therapy}

Cancer-directed therapy of the entire cohort

Chemotherapy

Immunotherapy

Anti-CD-20 antibody therapy

Anti-CD-38 antibody therapy

Immunomodulator

Proteasome inhibitor

Anti-VEGF antibody therapy

Anti-EGFR antibody therapy

Antibody-drug conjugate

Anti-HER antibody

Tyrosine kinase inhibitor

Bispecific T-cell engager

Androgen receptor-targeted therapy

PARP inhibitor

TGF- $\beta$ therapy

BTK inhibitor

BCL2 inhibitor

IDH1 inhibitor

CDK $4 / 6$ inhibitors

mTOR inhibitor

Endocrine therapy

Stem cell transplant

CAR-T/cellular therapy

Cancer-directed therapy within $90 \mathrm{~d}$ of COVID test

Chemotherapy

Immunotherapy

Anti-CD-20 antibody therapy

Anti-CD-38 antibody therapy

Immunomodulator

Proteasome inhibitor

Anti-VEGF antibody therapy

Anti-EGFR antibody therapy

Antibody-drug conjugate

Anti-HER antibody

Tyrosine kinase inhibitor

BiTE

Androgen receptor-targeted therapy

PARP inhibitor

TGF- $\beta$ therapy

BTK inhibitor

BCL2 inhibitor

IDH1 inhibitor

CDK 4/6 inhibitors

mTOR inhibitor

Endocrine therapy

Stem cell transplant

CAR-T/cellular therapy

Supportive care

Treated at outside institution (details unknown)

Table 2 summarizes frequencies of cancer-directed therapies in the overall cohort and within $90 \mathrm{~d}$ of COVID test ( $n=$ number of patients). our analysis, we observed a significant association between previous use of anti-CD20 therapy and SARS-CoV-2 IgG. A total of 17 patients had received anti-CD20 therapy, of which 7 patients had a negative SARS-CoV-2 IgG (Fisher's exact test, OR 0.09; $P=0.00013$ ). A similar finding was observed in the cohorts of patients who had a history of stem cell transplant. Ten patients had received a stem cell transplant in our cohort of which, four remained negative for SARS-CoV-2 IgG (Fisher's exact test, OR 0.1; $P=0.0057$ ). The above ORs refer to comparisons with the entire cohort of patients with cancer. The $P$ values are not adjusted for multiple hypothesis testing; however, false discovery rate (FDR) correction is provided for variables with more than two levels of analysis in Supplementary Tables 9, 11 and 12 . We also noted reduced seroconversion in patients who received CAR-T-cell therapy (33\%) and BiTE therapy (0\%); however, given the small number of patients in these cohorts, statistical analysis was not performed.

In contrast, we observed very high seroconversion rates in patients who received immunotherapy or endocrine therapy. Seventeen patients received previous immunotherapy for their cancer and all 17 of them manifested a positive SARS-CoV-2 antibody response. Despite this $100 \%$ seroconversion rate, the OR did not reach statistical significance $(P=0.38)$, likely due to high baseline frequency of seroconversion for the entire patient population. Of 71 patients who received endocrine therapy for their cancer, $70 \mathrm{mani}-$ fested a positive SARS-CoV-2 IgG (Fisher's exact test, OR 8.6 $=0.01$ compared to those without endocrine therapy). These results are summarized in Table 3.

The above results indicate that patients with hematological malignancies, anti-CD-20 therapy, CAR-T-cell therapy and stem cell transplant are associated with reduced seroconversion in patients with SARS-CoV-2. On the other hand, endocrine therapy has a strong association with positive antibody response in patients with SARS-CoV-2.

Active cancer and treatment within 90 days of SARS-CoV-2 IgG. We aimed to investigate potential confounders in the differential seroconversion rates noted for solid and hematological malignancies. We identified cancer status and cancer-directed therapy received within $90 \mathrm{~d}$ as potential key confounders and performed a multivariate logistic regression analysis. These variables were chosen as these would be biologically plausible to have an effect on seroconversion. Results indicate that the association between solid versus hematological malignancy and SARS-CoV-2 remain significant after accounting for active cancer and active cancer-directed treatment in the preceding $90 \mathrm{~d}$ of the COVID test, OR 4.004, $P=0.0026$ (Table 3 and Supplementary Table 10).

Association of steroid use and SARS-CoV-2 IgG test. We investigated baseline use of corticosteroids in our cohort. Twenty-three percent (61 of 261) of patients had steroid exposure before the SARS-CoV-2 test. Of these, 40 patients had occasional steroid use, whereas 21 patients had daily steroid use. Ten additional patients received steroids for COVID-19 infection. In a univariate analysis, steroid use at baseline showed a notable trend with lack of seroconversion $(P=0.06)$. Similarly, while the low numbers limit strong conclusions, steroid use for COVID-19 management also showed an association with absent seroconversion. The indications, frequencies and results of this analysis are summarized in the supplement.

Persistent SARS-CoV-2 PCR positivity. Eighteen percent (47 of 261) of patients underwent serial SARS-CoV-2 PCR testing as per institutional policies to document clearance of infection (Fig. 1). Thirty-five patients had a solid malignancy and 12 had hematological malignancy. The mean shedding time, calculated as the time between first and last positive SARS-CoV-2 PCR, was significantly higher in patients with hematological than in patients with solid 
Table 3 | Associations of therapies and cancer types with SARS-CoV-2 IgG positivity

Results $^{\mathrm{a}}$

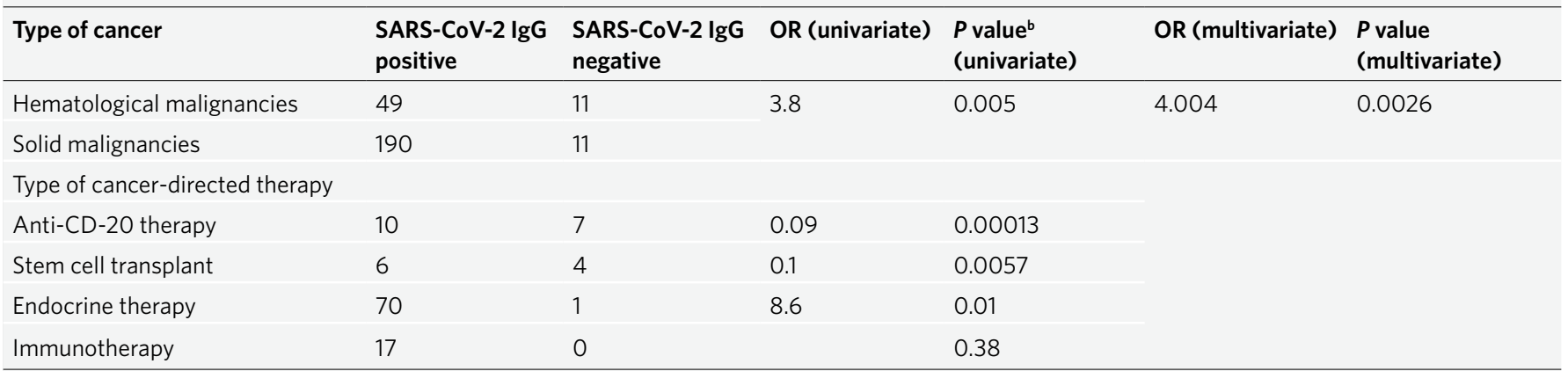

anivariate analysis was carried out between all treatment categories and seroconversion, only the statistically significant results are shown here. Univariate statistical test used was a two-sided Fisher's test and multivariate test was a two-sided Wald test on a logistic model. ${ }^{\circ} P$ values are uncorrected for multiple hypothesis testing and FDR correction is provided for variables where more than two levels of analysis was performed in Supplementary Tables 9,11 and 12 .

malignancies (61 d versus $33 \mathrm{~d}, P=0.007$; Table 4 ). Seropositivity was noted in 31 patients with a solid malignancy and 9 with a hematological malignancy. The remaining four patients with a solid malignancy and three with a hematological malignancy remained seronegative (Table 4). This observation again stresses the importance of close follow-up and monitoring of patients with hematological malignancies and may be impactful in designing quarantine strategies for these patients after clinical improvement from acute COVID-19 illness.

Serial SARS-CoV-2 IgG testing. Fifty-six patients underwent serial SARS-CoV-2 IgG testing. Of these, 53 had an initial positive test and 3 patients had an initial negative test. We collected data for time between first and last test available in our system. In this cohort, 44 of 53 patients remained persistently positive, whereas 9 patients turned seronegative. Eight of nine patients had a solid malignancy and one had a hematological malignancy. Seven of nine patients received treatment for cancer in the $90 \mathrm{~d}$ preceding a COVID test. Of the three initial patients who were seronegative, one patient turned seropositive and two remained persistently seronegative. The median time between first and last test in this cohort was $49 \mathrm{~d}$ (Supplementary Table 13).

Outcomes. Twenty-nine of 261 patients had died by the time of data cutoff date. Among them, 14 died due to progressive malignancy and 1 died of sequelae of COVID-19 infection (Supplementary Table 14).

\section{Discussion}

COVID-19 disease caused by SARS-CoV-2 has now affected more than 68 million humans worldwide, including over 27 million in the United States and has caused more than 400,000 deaths in the United States alone (Johns Hopkins Coronavirus Resource Center as of 5 February 2021). Older age and having multiple comorbid conditions have been identified as predictors of mortality in this disease $^{9}$. Several observational cohorts have identified that patients with cancer have a longer, protracted course with COVID-19 necessitating hospitalization and intensive care. Patients with hematological malignancies have been reported in many series, including our own, to have higher mortality compared to solid malignan$\operatorname{cies}^{6,10}$. While it was hypothesized in many cohorts that a diagnosis of cancer predicts mortality, data on this particular aspect are still evolving as recent matched studies report similar mortality in patients with cancer compared to age-matched controls without a diagnosis of cancer ${ }^{11}$. Nevertheless, concern about seroconversion in this patient population, which often receives immunosuppressive
Table 4 | Shedding time and associations with malignancy type

\begin{tabular}{llll}
$\begin{array}{l}\text { Type of } \\
\text { malignancy }\end{array}$ & Hematological malignancy & Solid malignancy & P value \\
\hline $\begin{array}{l}\text { SARS-CoV-2 } \\
\text { PCR shedding }\end{array}$ & $61 \mathrm{~d}$ & $33 \mathrm{~d}$ & 0.007 \\
time (mean) & & \\
$\begin{array}{l}\text { Number of } \\
\text { patients }\end{array}$ & 12 & 35 & \\
$\begin{array}{l}\text { SARS-CoV-2 } \\
\text { IgG test }\end{array}$ & Hematological malignancy & Solid malignancy & \\
$\begin{array}{l}\text { SARS-CoV-2 } \\
\text { IgG positive }\end{array}$ & 9 & 31 \\
$\begin{array}{l}\text { SARS-CoV-2 } \\
\text { IgG negative }\end{array}$ & 3 & & \\
\hline
\end{tabular}

Statistical test used is analysis of variance chi-squared test (sidedness is not applicable). Data were collected using Microsoft Excel and analyzed using R 3.6.2 (Methods).

treatments, has been raised as mounting a humoral immunity is crucial not only in recovery from the infection, but to also establish and maintain herd immunity through effective vaccination strategies.

This large cancer cohort reports seroconversion rates following SARS-CoV-2 infection. Ninety-two percent of patients manifested a positive antibody response in our study that was focused on a large cohort of ethnically diverse patients who survived SARS-CoV-2 infection. With the same SARS-CoV-2 IgG assay, seroconversion rates in the general population have been reported as $90-100 \%{ }^{12-14}$. Indeed, in an unselected cohort of 1,008 patients with SARS-CoV-2 PCR positivity in our health system who had subsequent antibody testing, the seroconversion rate was $91 \%$, nearly identical to the overall seroconversion rate of our cohort of patients with cancer, providing reassurance that most patients with cancer are able to mount an antibody response to SARS-CoV-2 similar to the general population (Supplementary materials).

In an observational study from Spain involving 43 patients with cancer, seroconversion was noted in $83 \%$ patients and was absent in $17 \%$ (6) patients. Four of the six patients were on immunosuppressive therapy, of which two received rituximab and two received cisplatin-based therapy ${ }^{15}$. Studies comparing seroconversion in patients with cancer versus controls, report seroconversion rates ranging from $72.5 \%{ }^{16}$ (retrospective) to $87.9 \%$ (prospective) ${ }^{17}$. A similar finding in an anti-CD20-treated patient was noted in a recently published case report ${ }^{18}$. 
In our study, we observed significant and clinically meaningful differences in seroconversion rates in patients who had received anti-CD20 therapy and stem cell transplants. The biological basis of this can be explained by the fact that anti-CD-20 therapy, such as rituximab, depletes native $\mathrm{B}$ cells not only in lymphoid tissue but also in bone marrow ${ }^{19}$. A statistically significant association was also seen with CAR-T-cell therapy (Supplementary Material) and BiTE therapy; however, given the small sample size, further validation in prospective cohorts is needed. CAR-T cells directed toward CD-19 also deplete native B cells, leading to hypogammaglobulinemia, often needing intravenous immunoglobulin replacement ${ }^{20}$. Patients who are recipients of stem cell transplantation are often subject to myeloablative doses of chemotherapy and total body irradiation, which contributes to profound immunosuppression in these patients. Our study in conjunction with existing literature highlights that patients with hematological malignancies who have received the aforementioned therapies, will need close follow-up and monitoring to document clearance of infection. Among these patients, seroconversion might not occur, possibly raising the concern of recurrent infections. As vaccines against SARS-CoV-2 are planned to be distributed on a large scale, monitoring SARS-CoV-2 IgG, immunoglobulin levels and lymphocyte subsets may be warranted in this patient population. Booster dosing may need to be studied in future trials and considered for this patient population should initial antibody responses be blunted.

Encouragingly, our study demonstrated high rates of seroconversion in patients with solid malignancies, in particular those who received immunotherapy and endocrine therapy for cancer treatment. Immunotherapy continuation has been specifically raised as a concern for patients with COVID-19 as immune-mediated pneumonitis is a significant side effect. Moreover, immunotherapy, specifically among patients with lung cancer, was associated with increased risk of ICU admission in one series of 275 patients ${ }^{21}$. On the contrary, two large cohorts, the UK Coronavirus Cancer Monitoring Project and the COVID-19 and Cancer Consortium (CCC 19) reported that mortality was not affected in patients with cancer and COVID-19 by type of anticancer therapy, including immunotherapy ${ }^{4,22}$. It is also hypothesized that immune-checkpoint inhibitors may induce immunocompetence in patients infected with SARS-CoV-2 (ref. ${ }^{23}$ ) based on previous data from human immunodeficiency virus and immunotherapy and ongoing trials with nivolumab in patients with sepsis ${ }^{24,25}$. Our $100 \%$ seroconversion rate provides supportive evidence that immunotherapy is not deleterious and rather, may support the hypothesis of restoring immunocompetence in patients with COVID-19. In addition, we note strong trends toward inferior seropositivity rates among patients receiving steroid therapy both before SARS-CoV-2 infection as well as for the management of COVID-19.

It is worth highlighting that patients receiving endocrine therapy for their malignancy (mostly breast cancer and prostate cancer) typically tend to have limited or no other cancer therapy exposure and therefore, less immunosuppression. This may explain our observation of strong positive seroconversion in patients treated with endocrine therapy.

A subset of patients in our cohort underwent serial SARS-CoV-2 IgG testing at provider discretion and 9 of 53 patients who were initially seropositive turned seronegative over time. These observations clearly need further validation in a larger cohort. Notably, these may have implications for guidelines and possible advocacy for continued vaccination of this vulnerable population.

Asymptomatic infection has been identified as a important factor in the community spread of SARS-CoV-2, which in turn, continues to propagate the pandemic ${ }^{26}$. As discussed previously, patients with cancer are prone to more symptomatic and serious illness. However, in our cohort, we found a surprisingly high rate of asymptomatic infections, with a higher frequency noted among patients with solid tumors. This finding is logical as patients with hematological malignancies are known to be prone to more serious illness and poorer outcomes. Many patients in our cohort tested positive as part of routine screening before procedures or during admission for unrelated acute problems. In some cases, patients who had contact with family members who were symptomatic with COVID-19 remained asymptomatic themselves. In a recent small study, seroconversion was noted in patients with cancer only if they had a symptomatic infection ${ }^{27}$. In our cohort $41 \%$ patients were defined as asymptomatic (114 of 261). Of these, 108 had positive SARS-CoV-2 IgG (Supplementary Table 4) and 6 had negative SARS-CoV-2 IgG. This finding suggests that asymptomatic infection also leads to seroconversion in the majority of cases and possibly contributes to expansion of the pandemic and herd immunity.

Another notable finding noted in our study is the tendency toward more persistent shedding of SARS-CoV-2 in patients with hematological malignancies, with a mean of $61 \mathrm{~d}$ despite clinical improvement in many cases. While we are unable to confirm whether virus was live in each patient, our findings seem concordant with a recent study that reported patients who had received stem cell transplant and CAR-T-cell therapy shed viable virus for up to 2 months from onset of symptoms ${ }^{28}$.

Our study has a few limitations warranting discussion, including its retrospective design and a small cohort among patients who received specific therapies that predicted seroconversion, calling for further validation in larger cohorts focused on these unique associations. Another limitation of the study may be a slight overestimation of the asymptomatic infection rate given the manner of asymptomatic infection needed to be defined in a retrospective design. Our cohort also represents standard of care practice wherein testing was performed at provider discretion and not as part of a prospective, controlled study; however, as PCR negativity was required for patients to be able to resume cancer management in our practices, testing was frequent in the majority of patients.

In summary, we present a large cohort of patients with malignancy who underwent SARS-CoV-2 IgG testing. Statistically significant absent seroconversion was observed in patients with hematological malignancies, patients receiving anti-CD-20 therapy, CAR-T-cell therapy and stem cell transplant. These findings may be impactful not only for clinical monitoring and surveillance, but also in designing and tailoring vaccination for this high-risk patient population. These findings should be investigated in larger, prospective studies for further validation but should provide immediate guidance for clinicians and researchers.

\section{Methods}

Study objectives. The primary objectives were to study the rate of seroconversion for SARS-CoV-2 IgG for patients with cancer and its association with type of malignancy and type of anticancer therapy. Additionally, we also aimed to study patterns in the natural history of COVID-19 and patients with cancer. Specifically, we studied the rate of symptomatic and asymptomatic infection in patients with cancer and COVID-19 and its association with type of malignancy and treatment received.

Study design. This was a real-world, observational, retrospective exploratory cohort study of the entire pool of patients with a cancer diagnosis managed at our institution with the prespecified criterion of positivity of one COVID test without previous hypotheses testing/power analyses. We collected data on demographic variables (age, sex, cancer diagnosis), comorbidities (excluding cancer itself), SARS-CoV-2 IgG result, SARS-CoV-2 RT-PCR result, cancer treatment history, onset of symptoms of COVID-19, subsequent disease course, treatment setting, complications and outcomes. The data were extracted through a retrospective chart-level medical record review using Montefiore Medical Center's EPIC electronic health record system. All patient information was de-identified. The study was approved by the Institutional Review Board of Albert Einstein College of Medicine/MHS. Informed consent was waived by Montefiore-Einstein Institutional Review Board as this was a retrospective chart review study. The Institutional Review Board (IRB) at Montefiore-Einstein provided ethics oversight (IRB no. 2020-11814). 
Definitions. Asymptomatic infection. Patients were classified as having an asymptomatic infection if (1) there was clear documentation at the time of a positive SARS-CoV-2 test that patient had no symptoms; (2) if there was documentation at the time of a SARS-CoV-2 IgG test that patient had no symptoms; or (3) a test result of SARS-CoV-2 PCR or IgG was present in the patient's chart and documentation was unable to confirm that patient had any symptoms (symptoms unknown, as these patients could not have had more than minimal symptoms they were clustered with the asymptomatic cohort).

Active cancer. We noted patient's malignancy status within $90 \mathrm{~d}$ preceding a SARS-CoV-2 test. Patients were classified as having an active malignancy if it was their initial diagnosis, relapsed or progressive disease. Patients were classified as having an inactive malignancy if their cancer was in remission or if they carried a diagnosis that did not warrant therapy (such as monoclonal gammopathy of unknown significance).

COVID-19 test methods (assay). SARS-CoV-2 RT-PCR. Real-time RT-PCR for SARS-CoV-2 was performed on nasopharyngeal swabs collected in viral transport medium using one of three testing platforms. These included the Hologic Panther Fusion, Abbott m2000 and Cepheid GenXpert SARS-COV-2 assays. All testing was performed in accordance with manufacturer or laboratory emergency use authorization instructions. Each assay was designed to amplify two separate regions within the SARS-CoV-2 viral genome and one amplification control in a single multiplex reaction. The target regions of amplification differ by platform with Hologic amplifying two separate regions of ORF1a, Abbott amplifying RdRp and $\mathrm{N}$ genes and Cepheid amplifying portions of the $\mathrm{N}$ and $\mathrm{E}$ genes.

SARS-CoV-2 IgG test. IgG testing was performed using the Abbott SARS-COV-2 $\mathrm{IgG}$ assay, which has received emergency authorization from the US Food and Drug Administration. The assay is a high throughput chemiluminescent microparticle immunoassay designed to detect IgG antibodies to the nucleocapsid of SARS-CoV-2. Recombinant SARS-CoV-2 antigen is incubated with a patient serum or plasma sample. IgG in a patient's sample reacts with an anti-human IgG acridinium-labeled conjugate to produce a chemiluminescent reaction measured as relative light units (RLUs). The greater the IgG level, the higher the RLU value. This relationship is reflected in the calculated signal-to-cutoff $(\mathrm{S} / \mathrm{C})$ index produced upon comparing patient RLU to the assay calibrator. Positive results for $\mathrm{IgG}$ antibodies are determined when the $\mathrm{S} / \mathrm{C}$ is $\geq 1.4$

Statistics and reproducibility. Associations between pairs of variables were assessed with standard statistical procedures. In the case of two-level categorical variables, a Fisher's exact test was used. For a two-level categorical and one numerical variable, we used a two-sample Student's $t$-test and results were then re-analyzed by Wilcoxon testing. For a multilevel categorical and one numerical variable, an analysis of variance test was carried out and results re-tested by nonparametric Kruskal-Wallis rank-sum test. Pairings between a two-level and a multilevel categorical variable were summarized in a table where each row tests the association of a single multilevel category to the remaining categories, split by the two-level categories. We also performed a multivariate logistic regression analysis to account for key confounding variables, such as active cancer treatment and active versus inactive malignancy. Multiple hypothesis adjustments were not made for all analyses, however, to account for multiple testing; an FDR correction is provided in the supplement where more than two-level testing was performed. Statistical analyses were not performed on cohorts of fewer than five individuals given instability of results in such small groups. Data were collected using Microsoft Excel and all analyses were run in R software v.3.6.2.

Reporting Summary. Further information on research design is available in the Nature Research Reporting Summary linked to this article.

\section{Data availability}

Primary data will be made available from the corresponding authors upon request to protect patient privacy. Data availability may be subject to consultation with and contingent of approval from the Montefiore-Einstein IRB.

\section{Code availability}

The utilized computer code has been deposited in GitHub (https://github.com/ kith-pradhan/CovidCancerReport). All analyses were conducted with built-in and freely available R packages. Further information on research design is available in the Nature Research Reporting Summary linked to this article.

Received: 12 December 2020; Accepted: 4 March 2021; Published online: 22 March 2021

\section{References}

1. Liu, J. et al. Clinical outcomes of COVID-19 in Wuhan, China: a large cohort study. Ann. Intensive Care 10, 99 (2020).
2. Guan, W.-j et al. Clinical Characteristics of Coronavirus Disease 2019 in China. N. Engl. J. Med. 382, 1708-1720 (2020).

3. Zhang, L. et al. Clinical characteristics of COVID-19-infected cancer patients: a retrospective case study in three hospitals within Wuhan, China. Ann. Oncol. 31, 894-901 (2020).

4. Kuderer, N. M. et al. Clinical impact of COVID-19 on patients with cancer (CCC19): a cohort study. Lancet 395, 1907-1918 (2020).

5. Dai, M. et al. Patients with cancer appear more vulnerable to SARS-CoV-2: a multicenter study during the COVID-19 outbreak. Cancer Discov.10, 783-791 (2020).

6. Mehta, V. et al. Case fatality rate of cancer patients with COVID-19 in a New York hospital system. Cancer Discov. 10, 935-941 (2020).

7. Saini, K. S. et al. Mortality in patients with cancer and coronavirus disease 2019: a systematic review and pooled analysis of 52 studies. Eur. J. Cancer 139, 43-50 (2020).

8. Jørgensen, T. L. et al. Comorbidity in elderly cancer patients in relation to overall and cancer-specific mortality. Br. J. Cancer 106, 1353-1360 (2012).

9. Du, R.-H. et al. Predictors of mortality for patients with COVID-19 pneumonia caused by SARS-CoV-2: a prospective cohort study. Eur. Resp. J. 55, 2000524 (2020).

10. Lee, L. Y. W. et al. COVID-19 prevalence and mortality in patients with cancer and the effect of primary tumour subtype and patient demographics: a prospective cohort study. Lancet Oncol. 21, 1309-1316 (2020).

11. Brar, G. et al. COVID-19 severity and outcomes in patients with cancer: a matched cohort study. J. Clin. Oncol. 38, 3914-3924 (2020).

12. Bryan, A. et al. Performance characteristics of the Abbott Architect SARS-CoV-2 IgG assay and seroprevalence in Boise, Idaho. J. Clin. Microbiol. https://doi.org/10.1128/JCM.00941-20 (2020).

13. Manalac, J. et al. Evaluation of Abbott anti-SARS-CoV-2 CMIA IgG and Euroimmun ELISA IgG/IgA assays in a clinical lab. Clin. Chim. Acta 510, 687-690 (2020).

14. Chew, K. L. et al. Clinical evaluation of serological IgG antibody response on the Abbott Architect for established SARS-CoV-2 infection. Clin. Microbiol. Infect. 26, 1256.e9-1256 (2020).

15. Garde-Noguera, J. et al. Impact of SARS-CoV-2 infection on patients with cancer: retrospective and transversal studies in Spanish population. Cancers https://doi.org/10.3390/cancers12123513 (2020).

16. Liu, T. et al. Low prevalence of IgG antibodies to SARS-CoV-2 in cancer patients with COVID-19. Int. J. Cancer 147, 3267-3269 (2020).

17. Marra, A. et al. Seroconversion in patients with cancer and oncology health care workers infected by SARS-CoV-2. Ann. Oncol. https://doi.org/10.1016/j. annonc.2020.10.473 (2020).

18. Yasuda, H. et al. Persistent COVID-19 pneumonia and failure to develop anti-SARS-CoV-2 antibodies during rituximab maintenance therapy for follicular lymphoma. Clin. Lymphoma Myeloma Leuk. 20, 774-776 (2020).

19. Houot, R. et al. Could anti-CD20 therapy jeopardise the efficacy of a SARS-CoV-2 vaccine? Eur. J. Cancer 136, 4-6 (2020).

20. Brudno, J. N. \& Kochenderfer, J. N. Toxicities of chimeric antigen receptor T cells: recognition and management. Blood 127, 3321-3330 (2016).

21. Robilotti, E. V. et al. Determinants of COVID-19 disease severity in patients with cancer. Nat. Med. 26, 1218-1223 (2020).

22. Lee, L. Y. et al. COVID-19 mortality in patients with cancer on chemotherapy or other anticancer treatments: a prospective cohort study. Lancet 395, 1919-1926 (2020).

23. Vivarelli, S. et al. Cancer management during COVID-19 pandemic: is immune checkpoint inhibitors-based immunotherapy harmful or beneficial? Cancers https://doi.org/10.3390/cancers12082237 (2020).

24. Day, C. L. et al. PD-1 expression on HIV-specific T cells is associated with T-cell exhaustion and disease progression. Nature 443, 350-354 (2006).

25. Hotchkiss, R. S. et al. Immune checkpoint inhibition in sepsis: a phase $1 \mathrm{~b}$ randomized study to evaluate the safety, tolerability, pharmacokinetics, and pharmacodynamics of nivolumab. Intensive Care Med. 45, 1360-1371 (2019).

26. Nikolai, L. A. et al. Asymptomatic SARS coronavirus 2 infection: invisible yet invincible. Int. J. Infect. Dis. 100, 112-116 (2020).

27. Fuereder, T. et al. SARS-CoV-2 seroprevalence in oncology healthcare professionals and patients with cancer at a tertiary care centre during the COVID-19 pandemic. ESMO Open https://doi.org/10.1136/ esmoopen-2020-000889 (2020).

28. Aydillo, T. et al. Shedding of viable SARS-CoV-2 after immunosuppressive therapy for cancer. N. Engl. J. Med. https://doi.org/10.1056/NEJMc2031670 (2020).

\section{Acknowledgements}

We acknowledge Cancer Center grant P30 CA013330 and NCORP grant 2UG1CA189859-06 in providing funding for this project. This work was supported partly by the Jane A. and Myles P. Dempsey fund and the Pelka Family fund. The 
funders had no role in study design, data collection and analysis, decision to publish or preparation of the manuscript.

\section{Author contributions}

The author contributions were as follows: conception, A.T., A.V., S.G. and B.H.; data collection, A.T., S.J., Z.C. and B.R.; statistical analysis, K.P.; and manuscript writing, A.T., A.P.S., S.P., R.A.S., J.S., D.Y.G., A.V., S.G. and B.H. All authors contributed to manuscript revisions and final approval.

\section{Competing interests}

The authors declare no competing interests.

\section{Additional information}

Supplementary information The online version contains supplementary material available at https://doi.org/10.1038/s43018-021-00191-y.

Correspondence and requests for materials should be addressed to S.G. or B.H.

Peer review information Nature Cancer thanks Fabrice Barlesi, Justin Gainor, and Samuel Rubinstein for their contribution to the peer review of this work.

Reprints and permissions information is available at www.nature.com/reprints. Publisher's note Springer Nature remains neutral with regard to jurisdictional claims in published maps and institutional affiliations.

(C) The Author(s), under exclusive licence to Springer Nature America, Inc. 2021 


\section{Reporting Summary}

Nature Research wishes to improve the reproducibility of the work that we publish. This form provides structure for consistency and transparency in reporting. For further information on Nature Research policies, see our Editorial Policies and the Editorial Policy Checklist.

\section{Statistics}

For all statistical analyses, confirm that the following items are present in the figure legend, table legend, main text, or Methods section.

$\mathrm{n} / \mathrm{a}$ Confirmed

$\bigotimes$ The exact sample size $(n)$ for each experimental group/condition, given as a discrete number and unit of measurement

$\bigotimes$ A statement on whether measurements were taken from distinct samples or whether the same sample was measured repeatedly

$\triangle$ The statistical test(s) used AND whether they are one- or two-sided

Only common tests should be described solely by name; describe more complex techniques in the Methods section.

$\bigotimes$ A description of all covariates tested

\ A description of any assumptions or corrections, such as tests of normality and adjustment for multiple comparisons

$\triangle$ A full description of the statistical parameters including central tendency (e.g. means) or other basic estimates (e.g. regression coefficient)

AND variation (e.g. standard deviation) or associated estimates of uncertainty (e.g. confidence intervals)

For null hypothesis testing, the test statistic (e.g. $F, t, r$ ) with confidence intervals, effect sizes, degrees of freedom and $P$ value noted Give $P$ values as exact values whenever suitable.

Х $\square$ For Bayesian analysis, information on the choice of priors and Markov chain Monte Carlo settings

$\bigotimes \square$ For hierarchical and complex designs, identification of the appropriate level for tests and full reporting of outcomes

$\bigotimes \square$ Estimates of effect sizes (e.g. Cohen's $d$, Pearson's $r$ ), indicating how they were calculated

Our web collection on statistics for biologists contains articles on many of the points above.

\section{Software and code}

Policy information about availability of computer code

Data collection Microsoft Excel

Data analysis R 3.6 .2

For manuscripts utilizing custom algorithms or software that are central to the research but not yet described in published literature, software must be made available to editors and

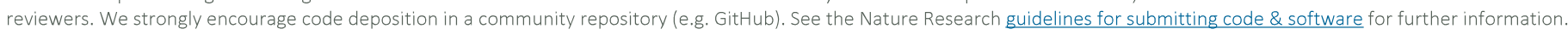

\section{Data}

Policy information about availability of data

All manuscripts must include a data availability statement. This statement should provide the following information, where applicable:

- Accession codes, unique identifiers, or web links for publicly available datasets

- A list of figures that have associated raw data

- A description of any restrictions on data availability

Primary data will be made available from the corresponding authors upon request to protect patient privacy. Data availability may be subject to consultation with and contingent of approval from the Montefiore-Einstein IRB.The utilized computer code has been deposited in github (https://github.com/kith-pradhan/ CovidCancerReport). All analyses were done with built-in and freely available R packages. 
Please select the one below that is the best fit for your research. If you are not sure, read the appropriate sections before making your selection.

\ Life sciences

Behavioural \& social sciences Ecological, evolutionary \& environmental sciences

For a reference copy of the document with all sections, see nature.com/documents/nr-reporting-summary-flat.pdf

\section{Life sciences study design}

All studies must disclose on these points even when the disclosure is negative.

Sample size $\begin{aligned} & \text { Patients who had a SARS-CoV-2 IgG test and a diagnosis of cancer were included in the analysis. The sample size was arrived at after filtering } \\ & \text { patients who were seen at Montefiore Einstein Center for Cancer Care for a SARS-CoV-2 IgG test. Please refer to consort diagram of the } \\ & \text { manuscript for details. A sample size of } 261 \text { was deemed adequate as this met our inclusion criteria. }\end{aligned}$
Data exclusions
Replication natients were excluded if age <18 and if they did not have a diagnosis of cancer and a SARS-CoV-2 IgG test
Randomization not applicable (retrospective chart review only)
Blinding not applicable (retrospective chart review only)
$\begin{aligned} & \text { not applicable (retrospective chart review only). Blinding was not relevant to our study as no experimental test or treatment was } \\ & \text { administered. }\end{aligned}$

\section{Reporting for specific materials, systems and methods}

We require information from authors about some types of materials, experimental systems and methods used in many studies. Here, indicate whether each material, system or method listed is relevant to your study. If you are not sure if a list item applies to your research, read the appropriate section before selecting a response.

\begin{tabular}{|c|c|}
\hline$n / a$ & Involved in the study \\
\hline$凶$ & $\square$ Antibodies \\
\hline Х & $\square$ Eukaryotic cell lines \\
\hline Х & $\square$ Palaeontology and archaeology \\
\hline$\bigotimes$ & $\square$ Animals and other organisms \\
\hline \begin{tabular}{|c} 
\\
\end{tabular} & $\bigotimes$ Human research participants \\
\hline Х & $\square$ Clinical data \\
\hline 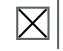 & $\square$ Dual use research of concern \\
\hline
\end{tabular}

\begin{tabular}{l|l} 
Methods \\
\hline n/a & Involved in the study \\
$\square$ & $\square$ ChIP-seq \\
$\searrow$ & $\square$ Flow cytometry \\
$\triangle$ & $\square$ MRI-based neuroimaging
\end{tabular}

\section{Human research participants}

\section{Policy information about studies involving human research participants}

Population characteristics

Recruitment

Ethics oversight
Adult cancer patients who received care at Montefiore Medical Center with a SARS-CoV-2 IgG test were included in the study. This is a retrospective study. Median age of the cohort is 64 years (range 20-90). Fifty-one percent patients are female and $49 \%$ are male. Any active or past diagnosis of cancer was included and all treatment received by each patient was recorded.

Study participants were not recruited, only retrospective chart review was performed. Informed consent was waived by Montefiore-Einstein institutional review board as this is a retrospective chart review study

Institutional review board at Montefiore medical center provided ethics oversight. IRB \# 2020-11814 